\title{
Portable monitoring in sleep apnea
}

\author{
Thomas Penzel • Alexander Blau • Carmen Garcia • \\ Christoph Schöbel • Michaela Sebert • Ingo Fietze
}

Published online: 8 March 2012

(C) Springer Science+Business Media, LLC 2012

\begin{abstract}
Portable sleep apnea monitoring or home testing for sleep-disordered breathing focuses on recent developments of these powerful diagnostic tools. Evidence-based reviews and innovative single studies with specific systems are considered. Systems become less intrusive and self applicable. Electrocardiogram-derived respiration, photoplethysmogram analysis, midsagittal jaw movements, and respiratory sound analysis are reviewed. Categories of systems with 4 to 6 channels and 1 to 3 channels are introduced and presented. The importance of a high pretest probability is elucidated. Open research questions regarding these systems are mentioned. Technological issues are not most important in this debate. The health economic aspects in using portable sleep apnea monitoring have to be considered as well. Portable monitoring of sleep apnea is probably less expensive than cardiorespiratory polysomnography and can help to overcome the limited availability of sleep lab-based diagnostic places. But by increasing the quantity of investigations it may cause additional costs too.
\end{abstract}

Keywords Sleep apnea $\cdot$ Sleep disordered breathing · Sleep related breathing disorders $\cdot$ Ambulatory diagnosis $\cdot$ Portable monitoring $\cdot$ Home recording $\cdot$ Heart rate $\cdot$ Heart rate variability - Electrocardiographic-derived respiration . Oxygen saturation $\cdot$ Photoplethysmography $\cdot$ Sleep recording $\cdot$ Polysomnography $\cdot$ Clinical trials $\cdot$ Review

T. Penzel $(\bowtie) \cdot$ A. Blau $\cdot$ C. Garcia $\cdot$ C. Schöbel $\cdot$ M. Sebert •

I. Fietze

Sleep Center, Charité Universitätsmedizin Berlin,

Charitéplatz 1,

10117 Berlin, Germany

e-mail: thomas.penzel@charite.de

\section{Introduction}

Sleep-related breathing disorders are recognized as having a high prevalence and causing remarkable cardiovascular risk. In order to diagnose disordered breathing during sleep a reliable diagnosis is required. The steps toward a reliable diagnosis are first a detailed sleep medicine interview, supported by a number of validated questionnaires for sleepiness, snoring, and witnessed breathing pauses, second a clinical investigation assessing BMI, blood pressure, and waist-hip circumference ratio, third portable sleep apnea monitoring, and only at the fourth and last step an attended cardiorespiratory polysomnography which is performed in a sleep laboratory or sleep center. Attended cardiorespiratory polysomnography is the reference standard for many sleep disorders where manifestations of events can be observed during the night. Cardiorespiratory polysomnography requires the recording of several physiological signals during a night under standardized conditions with the attendance of trained sleep medicine personnel. The completed recording is scored by a trained sleep technician and the summarizing report is validated by a sleep physician thereafter. This validated and approved approach is a time and resource consuming process.

In view of the high number of patients with sleep disorders there are too few sleep laboratory beds available to investigate all patients with a high likelihood to suffer from sleep apnea. Therefore strategies are developed to determine who should undergo a polysomnography and who not. Patients with a high pretest probability to suffer from sleep apnea need only portable monitoring to diagnose the disorder. Only if other comorbid disorders are present or if the results obtained by portable monitoring are not well interpretable then a cardiorespiratory polysomnography for clarification is indicated. Portable monitoring is becoming the 
method of choice for the primary assessment of sleepdisordered breathing $[1 \bullet]$.

Based on the diagnosis in the sleep center the appropriate treatment can be initiated. Usually the treatment is a chronic treatment due to the chronic nature of most sleep disorders. For sleep-disordered breathing the therapy of choice is home ventilation with nasal continuous positive airway pressure (CPAP). The nocturnal ventilation support is rather a physical aid than a causative cure. The effectiveness of the chronic therapy should be monitored over time at least once per year. Again diagnostic strategies are needed for this kind of treatment follow-up studies. The requirements for this kind of investigation are different from the initial diagnosis, because the disorder had been characterized already. So there is no need to check for other sleep disorders as long as no additional complaints are mentioned. The main purpose of the follow-up study is to track whether the initiated therapy is still effective. The titrated pressure may need adjustment. Changes in the type of sleep-disordered breathing may occur. There may be an increase of the percentage of central apnea events among the total number of apnea events. This may require a change of treatment modalities. Therefore, the treatment follow-up studies should include monitoring of respiratory parameters over the night and should be reviewed carefully. Portable monitoring is most appropriate for this kind of follow-up studies.

\section{Cardiorespiratory polysomnography as the diagnostic reference}

The basic tool and the reference method for sleep medicine diagnosis in the sleep laboratory is attended cardiorespiratory polysomnography. Cardiorespiratory polysomnography records physiological signals to quantify sleep and sleep disorders to achieve a diagnosis according to the International classification of sleep disorders revision 2 [2]. The basic rules were defined in a manual for the recording and evaluation of sleep [3]. This manual specifies the recording and evaluation of the electroencephalogram (EEG), the electrooculogram (EOG), and the submental electromyogram (EMG). A few years ago the manual had been expanded and revised to include the recording of respiration, cardio-circulatory functions, and movement parameters. Age-specific aspects such as pediatric sleep EEG and respiration in children were included in this revision too [4]. The new manual is based on a systematic literature review and a evidence evaluation [5-8].

Despite this new manual is now regarded as the reference standard for cardiorespiratory polysomnography the limitations of this approach should be considered when evaluating portable sleep studies which in general compare their results against this reference. Limitations apply to the final conclusion, the diagnosis of sleep-related breathing disorders [9]. We still do not know the value of an epoch-byepoch sleep staging using polysomnography in sleep apnea when predicting mortality or determining the cardiovascular risk in these patients. In addition, any threshold on a number of sleep apnea events expressed as an apnea-hypopnea index (AHI) is arbitrary and gives little insight into pathophysiology and the consecutive cardiovascular risk. The scoring of apnea and hypopnea events using alternative definitions results in different diagnoses [10] and a comparison of clinical relevance of these different diagnoses is missing. We do not fully understand the contributions of sleep fragmentation, arousals, intermittent hypoxia, and intrathoracic pressure changes to the pathophysiology and the subsequent cardiovascular risk associated with sleep-disordered breathing. We do understand that statistics for mortality and for health care economics do need thresholds in order to say that a disorder is present of absent. Still when judging about portable monitoring for the diagnosis of sleep-disordered breathing the uncertainty regarding the $\mathrm{AHI}$ as a measure of the ultimate accuracy and quality of the particular monitoring device must be kept in mind. Sometimes even simpler ways to identify patients with sleep-disordered breathing are feasible and not too bad in terms of clinical significance [11].

\section{Portable sleep apnea monitoring}

Portable monitoring of sleep-disordered breathing stands on the basis of cardiorespiratory polysomnography. The knowledge and the technology as well as recording and scoring criteria are derived from the above-described cardiorespiratory polysomnography. Multiple studies on individual systems and a considerable number of evidence-based reviews give evidence that ambulatory sleep recording devoted to sleep apnea is as specific and as reliable as sleep laboratory recordings [12-14]. Finally there are enough studies available to give evidence that portable monitoring of sleepdisordered breathing can be used when limitations are considered. Very recently a workshop consensus report presents the current view of medical societies involved (American Thoracic Society, American Academy of Sleep Medicine, American College of Chest Physicians, European Respiratory Society) with this diagnostic procedure and provides directions for further research [15••].

When discussing the technologies and recommendations of the studies, respectively the systems used, it has to be considered as a prerequisite that all systematic studies have been conducted on clinical populations [16, 17]. Clinical populations are different to the general population because they have been sent to the sleep center due to suspected sleep disorders. This selection is termed as a high pretest 
probability $[12,18]$. In order to understand a high pretest probability better, the complaints leading to this pretest probability are given here. The complaints are reported by the patient under consideration and can be supplemented by his or her bed partner.

1) Loud and irregular snoring

2) Observed or reported nocturnal cessation of breathing

3) Excessive daytime sleepiness

4) Unspecific mental problems such as fatigue, low performance, cognitive impairment

5) Movements during sleep

6) Morning dizziness, general headache, dry mouth

7) Impaired sexual functions

8) Obesity

9) Arterial hypertension, cardiac arrhythmias

A grading of the pretest probability would be very useful in order to quantify who might be more likely to suffer from sleep apnea or even from a more severe sleep apnea. This has not been investigated in clinical practice [19].

A Pubmed research on December 31, 2011 with the term "portable sleep monitoring" resulted in 360 references with 44 from the year 2011. A Pubmed search on "sleep apnea testing" resulted even in 640 references with 66 from the year 2011. A few publications present new devices and comparative studies of the new devices with polysomnography. A few papers present reviews on existing devices. The majority of papers discuss the role of portable sleep apnea monitoring in the diagnostic process of sleep apnea diagnosis. Some of the papers discuss the management of patients with sleep apnea in general and others focus on portable monitoring. As a result, the next paragraphs will give first a short technical overview on current and new systems. Then the role of portable sleep apnea monitoring is discussed considering the requirements and concerns mentioned above.

\section{Ambulatory recording of sleep-disordered breathing with 4 to 6 channels}

Systems for the diagnosis of sleep-disordered breathing are divided into four categories defined in a standard of practice publication [20]. Most systems used for the diagnosis of sleep-disordered breathing record four to six physiological signals without sleep EEG. Evidence-based reviews committed by the health technology assessment agencies about these systems [14] revealed a limited reliability. The review reported up to $17 \%$ false-negative and between $2 \%$ and $31 \%$ false-positive findings of sleep apnea. These high error rates compared to polysomnography are not acceptable and Ross et al. [14] concluded that portable monitoring of sleep apnea is not recommended. A few years later studies with revised systems showed that the limited channel systems improved $[16,21]$. If systems use a thoughtful selection of physiological signals to be recorded, if they have a very good signal acquisition and a very good signal processing part then the number of false-positive diagnoses is reduced considerably [12]. If studies which are more dedicated to the general population are compared with studies with clinical populations, then the importance of a high pretest probability becomes clear. A high pretest probability reduces the number of false-positive diagnoses again. Altogether the specificity gets high enough to conclude that portable monitoring of sleep apnea can be recommended under certain conditions. The conditions are:

1) Systems should be only used by certified sleep physicians based in certified sleep centers. The sleep medicine education comprises the teaching of a comprehensive sleep medicine interview and assessment of complaints before an ambulatory recording is performed. This increases the pretest probability explained above.

2) Ambulatory monitoring of obstructive sleep apnea can be recommended if no other comorbid pulmonary, cardiovascular, mental, neurological, and neuromuscular disorders, or heart failure, or other sleep disorders is present. Other sleep disorders could be central sleep apnea, PLMD, insomnia, circadian sleep wake disorders, and narcolepsy.

3) According to published studies the ambulatory monitoring systems are not able to distinguish central and obstructive sleep apnea events.

4) Ambulatory systems for the diagnosis of sleep apnea need to record oronasal airflow by means of thermistor or nasal pressure sensors, respiratory effort by means of inductive plethysmography, oxygen saturation (with a short averaging period over few pulses, meaning three to six pulses), pulse or heart rate and body position.

5) The evaluation of the recordings should be a visual scoring of respiratory events and the rules should be the same as specified for polysomnography [4]. Editing of events is necessary in order to remove artifacts found during the recording period. Therefore the visual scoring should be done by trained personnel.

6) The technical specifications and sampling rates for the digital recording should be the same as specified in the evidence-based recommendation for cardiorespiratory polysomnography [4].

Today many different devices fulfill these requirement. They all have a pulse oximeter to record oxygen saturation and pulse rate. Most systems record oronasal airflow with nasal pressure. Some systems still use thermistors for flow recording. Most systems record respiratory effort by either piezo sensors or inductive plethysmography. Some systems use just one belt for ribcage movements whereas others used 
two belts with abdominal movements recording in addition. All systems record body position. Few systems record ECG and ECG-derived heart rate. Most systems offer options to record pressure at a mask used for ventilation therapy. This is an essential feature in order to use the ambulatory device for treatment follow-up studies, as mentioned earlier. Some systems allow to record additional EMG tibialis to diagnose leg movements. But there have been no systematic studies on this kind of option. It remains an open question whether this portable monitoring is a sufficient diagnosis of PLMD. Few systems can add EEG channels to record sleep EEG. No systematic studies were performed to evaluate this option and the added value of a validated sleep duration. Many of the systems had been validated with their basic signal setup together with their scoring and analysis software. As a general conclusion for these studies all systems show good performance with some minor differences. No general preference to one or another system can be given based on the studies.

\section{Ambulatory recording of sleep-disordered breathing with 1 to 3 signals}

Systematic reviews for the diagnosis of sleep-disordered breathing have revealed that systems with 1 to 3 channels (pulse oximetry, long-term ECG, actigraphy, oronasal airflow) result in too many false-negative (up to $17 \%$ ) and too many false-positive (up to $31 \%$ ) findings [14]. This result remained valid in the recent review by Collop et al. [12]. Therefore the application of these devices is not recommended for a definite diagnostic test for obstructive sleep apnea or to exclude the presence of obstructive sleep apnea.

However, some of these devices provide results in patients with severe sleep apnea that are valid and give clear hints for sleep-disordered breathing. Therefore recording of high quality achieved with validated systems of this category can be used to increase the pretest probability considerably before conducting a cardiorespiratory polysomnography or even before having a portable sleep apnea monitoring test.

Most technical innovations are currently made in this category of devices. It is challenging and rewarding to develop 1 to 3 channel devices which perform well and allow to diagnose sleep-disordered breathing in new patient groups and provide tools for the diagnosis of sleep apnea to physicians with little knowledge about sleep medicine. We believe that before therapy is initiated a physician with a profound knowledge about sleep-disordered breathing and with the different treatment options should review the case $[1 \bullet]$.

The following paragraphs will focus on new technologies applicable both for 1 to 3 channel and for 4 to 6 channel diagnostic devices.

\section{New methods for portable sleep apnea monitoring}

Different approaches are explored to develop new technologies for the diagnosis of obstructive sleep apnea. Some of these just try to develop new sensors in order to assess respiration and disturbed respiration during the night, others try to assess the cardiovascular risk of the patient or sleep with the pathology of sleep fragmentation.

\section{Assessment of respiration}

A couple of different new sensors use surrogate signals in order to derive respiratory effort noninvasively. Some of these devices try to derive respiration from some kind of directly respiration-related signals. These systems and concepts are highlighted in this section.

A first-line approach is the recording of respiratory airflow at the nose and the mouth. Usually this is used together with oxygen saturation. These simple screening devices provide a straight analysis for respiratory cessations. They even try to distinguish obstructive and central respiratory events by an analysis of flow limitation. Due to problems with obstructed nostrils, with partial breathing through the mouth, through blocked air tubes and other kinds of artifacts, the validity of this analysis and in general these systems is limited. Nevertheless there are good validation studies available [22, 23].

One approach tries to analyze respiratory sounds in order to derive noninvasive measures for increased respiratory effort from the chest [24]. In other systems respiratory sounds are recorded at the throat and signal processing separates cardiac and movement sound first from breathing sounds and snoring. Together with oximetry breathing is quantified and snoring is investigated to detect respiratory cessations $[25,26]$.

One other approach is the recording of midsagittal jaw movements based on magnetic distance determination [27]. A magnetic sensor on the chin and one on the forehead allow to determine continuously the relative jaw movements. From this it is possible to derive respiration and snoring. The analysis of respiration and snoring is then used to detect respiratory events to diagnose sleep apnea [28]. By further analysis it seems possible to estimated a sleep wakefulness profile $[29,30]$. Combined with pulse oximetry and perhaps a cardiovascular parameter this is a simple and promising device.

Pulse wave analysis

Many systems try to exploit the pulse wave on the finger or other peripheral sites and try to derive parameters from the pulse wave in order to assess cardiovascular risk. The pressure wave may be detected with the photoplethysmography 
used for oxygen saturation anyhow and can be used to detect all forms of respiratory events [31] and cardiovascular risk as associated with sleep apnea $[32,33]$.

Peripheral arterial tonometry [34] can be used to assess the cardiovascular risk by addressing endothelial function during sleep-disordered breathing. Arousals which accompany sleep apnea events are accompanied by attenuated pulse amplitude. This is due to a peripheral vasoconstriction caused by sympathetic tone activation. If pulse rate is analyzed in addition it is possible to distinguish slow wave sleep and REM sleep [35]. Several validation studies were published using the Watch-PAT in sleep apnea patients with very good results $[33,36,37]$.

\section{Assessment of ECG and heart rate variables}

ECG-derived variables are very attractive for a simple detection of sleep apnea due to low costs and wide availability. In order to detect sleep apnea using the ECG no additional electrodes nor additional devices would be needed. Only an additional evaluation software could do the analysis. This could be retrospectively using previous recorded data.

The analysis of the ECG for the detection of sleep-related breathing disorders can be based on the analysis of heart rate. Sleep apnea is accompanied by a cyclical variation of heart rate, described already many years ago [38]. Periodic changes in heart rate are related to the changes in sympathetic tone with apnea events [39]. Modern analysis of heart rate variability can satisfactorily derive cyclical variations of heart rate $[40,41]$. In addition the morphology of the ECG wave itself is modulated by respiration. The derived respiratory curve is called ECG-derived respiration [42] and correlates with respiratory effort. This can be used to detect sleep-disordered breathing [31, 43]. By combining ECGderived respiration and sleep apnea related heart rate variability a detection of sleep apnea is possible [44•].

\section{Assessment of ECG and oximetry}

A number of devices which use the ECG analysis techniques mentioned above try to link this to previous techniques. Already early in the portable diagnosis of sleep apnea pulse oximetry had been used. Pulse oximetry alone has large limitations in patients with arrhythmias or with additional lung diseases such as COPD. Combining ECG-based sleep apnea analysis and oximetry is therefore a very promising approach [45]. An early study of pulse rate in addition to oximetry [46] successfully improves the detection of sleep apnea. One retrospective study did show the advantage over pulse oximetry alone when using ECG analysis in addition [47]. In this study ECG from a parallel polysomnography was analyzed. Based on these results a combined long-term ECG recording system with oximetry was tested in a prospective study and provided very convincing results in terms of sleep apnea detection [45].

\section{The role of portable sleep apnea monitoring}

Many new studies do show the high reliability in detecting sleep apnea with portable monitoring devices [23]. Many of the open research questions which are needed to clarify the conditions and restrictions for the use of portable monitoring are now addressed in the recent studies $[12,15 \cdot \bullet]$. The important conditions are no longer technical limitations but often study limitations such as a preselected group of patients. This corresponds to the high pretest probability. And this prohibits the use of portable monitoring as a screening tool for subjects where we would like to exclude sleep apneas such as professional drivers of people with supervision tasks.

We do see that diagnostic approaches and therapy approaches differ much between countries worldwide [48]. We see that the development of sleep medicine in some countries is very advanced thinking about strategies where to go $[49 \cdot \bullet, 50]$ and may be very basic with just having portable monitoring for sleep apnea available [48]. To reconsider the role of sleep medicine and to develop strategies for diagnostic procedures and therapy of patients under increased health risk [49] can be used for all countries and can help to identify and help all patients suffering from sleep-disordered breathing. In Germany we started a debate on different levels of sleep medicine service which would include different levels of medical expertise and correspondingly different levels of equipment complexity. Only few competence centers would have research, training, and all technical and knowledge competence [50]. Many community-based centers may have basic sleep medicine knowledge and just portable diagnostic tools.

The other issue is health economy and patient care. Still we do not know the threshold regarding sleep apnea severity: How many apneas, which duration of apnea events, how much sleep fragmentation, or which degree of hypoxia presents a substantial cardiovascular risk with increased mortality. Which increase in mortality will justify a treatment with CPAP or another life-long therapy? In view of limited therapy adherence how strict should we be with treatment follow-up studies? All diagnostic tests can be done easily with portable monitoring. And therefore the economic decisions are now key for the development of new strategies for the management of sleep apnea.

Patients may be diagnosed and even treated at home. One outcome study did show that the 4-week outcome in sleepiness and CPAP adherence is similar to sleep lab based diagnosis and treatment [51•]. A limitation of the study is that the follow-up was obtained after 4 weeks only and that 
patients still have a very high motivation after that short period [52]. Motivation to use CPAP may decline more at home. Still this needs to be studied.

\section{Conclusions}

Although attended cardiorespiratory polysomnography is the reference standard for the diagnosis of disordered breathing during sleep, evidence-based literature research has led to the conclusion that a diagnosis of obstructive sleep apnea can be performed using portable methods under certain conditions. The recording must consist of oxygen saturation, airflow, respiratory effort, heart or pulse rate and body position. It is not possible to distinguish central and obstructive respiratory events. Portable monitoring is reliable if it is performed under the supervision of personnel trained for sleep medicine and if the subjects investigated have a high pretest probability to suffer from sleepdisordered breathing. In addition the subjects investigated should not suffer from other sleep disorders and comorbid disorders such as heart failure, stroke, diabetes mellitus, obstructive or restrictive lung diseases, or severe cardiac arrhythmias.

Systems with fewer channels can give good indications for sleep-disordered breathing but are not sufficient for diagnostic purposes. It is expected that advances will push the border between "good indications to suffer" and "sufficient and reliable diagnosis" of disordered breathing during sleep more toward simpler systems and approaches. In order to prove the advanced usefulness of new technologies good clinical studies have to be published and these must have sufficient size and sufficient reference technology to compare with.

Technology advances need to be accompanied by economy-driven strategies to diagnose and treat patients with sleep apnea. Recent approaches to diagnose patients at home and even treat patients at home seem to provide similar effectiveness in terms of outcome as sleep laboratory based studies. Economically good home-based studies may be more feasible than sleep laboratory based studies when we consider the high prevalence of the disorders and the still unmet clinical needs to recognize and treat patients with sleep-disordered breathing.

Acknowledgment Itamar has provided consumables to T. Penzel to conduct research protocols with the Watch-PAT device. Neuwirth Medical has provided equipment to the sleep center for studies. Weinmann $\mathrm{GmbH}$ has provided equipment to the sleep center for research studies.

Disclosure T. Penzel: owns shares of advanced sleep research $\mathrm{GmbH}$, Somnico $\mathrm{GmbH}$, The Siestagroup GmbH. Received grants from Breas, Hoffrichter, Philips/Respironics, Resmed, Weinmann, Apnex, Hypnocore; A. Blau: none; C. Garcia: none; C. Schöbel: none;
M. Sebert: grant from Philips/Respironics and speakers' bureau for UCB; I. Fietze: none.

\section{References}

Papers of particular interest, published recently, have been highlighted as:

- Of importance

•- Of major importance

1. • Penzel T, Blau A, Schöbel C, Fietze I. Ambulatory diagnosis of OSA and new technologies. In: Sleep Apnoea. European Respiratory Monograph Nr. 50. Edited by McNicholas WT, Bonsignore MR. Sheffield: European Respiratory Society; 2011: 136-49. This review on recent developments in sleep laboratory technology and in technologies for portable sleep apnea monitoring has been the technical basis for the review presented here.

2. American Academy of Sleep Medicine. International classification of sleep disorders. Diagnostic and coding manual 2nd edn. American Academy of Sleep Medicine, Westchester, Ill. (2005).

3. Rechtschaffen A, Kales A. A manual of standardized terminology, techniques, and scoring system for sleep stages of human Subjects. US Department of Health, Education, and Welfare Public Health Service - National Institute of Health (1968)

4. Iber C, Ancoli-Israel S, Chesson A, and Quan SF for the American Academy of Sleep Medicine. The AASM Manual for the Scoring of Sleep and Associated Events: Rules, Terminology and Technical Specifications, 1st ed.: Westchester, Illinois: American Academy of Sleep Medicine, 2007

5. Silber MH, Ancoli-Israel S, Bonnet MH, Chokroverty S, GriggDamberger MM, Hirshkowitz M, et al. The visual scoring of sleep in adults. J Clin Sleep Med. 2007;3:121-31.

6. Penzel T, Hirshkowitz M, Harsh J, Chervin RD, Butkov N, Kryger $\mathrm{M}$, et al. Digital analysis and technical specifications. J Clin Sleep Med. 2007;3:109-20.

7. Redline S, Budhiraja R, Kapur V, Marcus CL, Mateika JH, Mehra $\mathrm{R}$, et al. The scoring of respiratory events in sleep: reliability and validity. J Clin Sleep Med. 2007;3:169-200.

8. Caples SM, Rosen CL, Shen WK, Gami AS, Cotts W, Adams M, et al. The scoring of cardiac events during sleep. J Clin Sleep Med. 2007;3:147-54.

9. Whitelaw WA, Burgess KR. Diagnosis of sleep apnoea: some critical issues. Indian J Med Res. 2010;131:217-29.

10. Ruehland WR, Rochford PD, O’Donoghue FJ, Pierce RJ, Singh P, Thornton AT. The new AASM criteria for scoring hypopneas: impact on the apnea hypopnea index. Sleep. 2009;32:150-7.

11. Mulgrew AT, Fox N, Ayas NT, Ryan CF. Diagnosis and initial management of obstructive sleep apnea without polysomnography: a randomized validation study. Ann Intern Med. 2007;146:157-66.

12. Collop NA, Anderson WM, Boehlecke B, Claman D, Goldberg R, Gottlieb DJ, et al. Clinical guidelines for the use of unattended portable monitors in the diagnosis of obstructive sleep apnea in adult patients. J Clin Sleep Med. 2007;3:737-47.

13. Collop NA. Portable monitoring for the diagnosis of obstructive sleep apnea. Curr Opin Pulm Med. 2008;14:525-9.

14. Ross SD, Sheinhait IA, Harrison KJ, et al. Systematic review and meta-analysis of the literature regarding the diagnosis of sleep apnea. Sleep. 2000;23:519-32.

15. • Kuna ST, Badr MS, Kimoff RJ, Kushida C, Lee-Chiong T, Levy P, et al. An official ATS/AASM/ACCP/ERS Workshop report: research priorities in ambulatory management of adults with obstructive sleep apnea. Proc Am Thorac Soc. 2011;8:1-16. This report summarizes 
the current debate on clinical usefulness of portable sleep apnea monitoring and gives advice for future research..

16. Flemons WW, Littner MR, Rowley JA, et al. Home diagnosis of sleep apnea: a systematic review of the literature. An evidence review cosponsored by the American Academy of Sleep Medicine, the American College of Chest Physicians, and the American Thoracic Society. Chest. 2003;124:1543-79.

17. Chesson AL, Berry RB, Pack A. Practice parameters for the use of portable monitoring devices in the investigation of suspected obstructive sleep apnea in adults. Sleep. 2003;26:907-13.

18. Mayer G, Fietze I, Fischer J, Penzel T, Riemann D, Rodenbeck A, et al. S3-Leitlinie Nicht erholsamer Schlaf/Schlafstörungen. Somnologie. 2009;13:4-160.

19. Ayas NT, Fox J, Epstein L, Ryan CF, Fleetham JA. Initial use of portable monitoring versus polysomnography to confiorm obstructive sleep apnea in symptomatic patients: An economic decision model. Sleep Medicine. 2010;11:320-4.

20. Standards of Practice Committee of the American Sleep Disorders Association. Practice Parameters for the use of portable recording in the assessment of obstructive sleep apnea. Sleep. 1994;17:372-7.

21. American Thoracic Society. Executive summary on the systematic review and practice parameters for portable monitoring in the investigation of suspected sleep apnea in adults. Am J Respir Crit Care Med. 2004;169:1160-3.

22. Ragette R, Wang Y, Weinreich G, Teschler H. Diagnostic performance of single airflow channel recording (apneaLink) in home diagnosis of sleep apnea. Sleep Breath epub before print (2009)

23. Oktay B, Rice TB, Atwood CW, Passero M, Gupta N, Givelber R, et al. J Clin Sleep Med. 2011;7:384-90.

24. Kaniusas E, Pfützner H, Saletu B. Acoustical signal properties for cardiac/respiratory activity and apneas. IEEE Trans Biomed Eng. 2005;52:1812-22.

25. Yadollahi A, Moussavi Z. Acoustic obstructive sleep apnea detection. Conf Proc IEEE Eng Med Biol Soc 2009;7110-13.

26. Yadollahi A, Giannouli E, Moussavi Z. Sleep apnea monitoring and diagnosis based on pulse oximetry and tracheal sound signals. Med Biol Eng Comput. 2010;48:1087-97.

27. Senny F, Destine J, Poirrier R. Midsagittal jaw movement analysis for the scoring of sleep apnea and hypopneas. IEEE Tran Biomed Eng. 2008;55:87-95.

28. Cheliout-Heraut F, Senny F, Djouadi F, Ouayoun M, Bour F. Obstructive sleep apnoea syndrome: comparison between polysomnography and portable sleep monitoring based on jaw recordings. Neurophysiol Clin. 2011;41:191-8.

29. Senny F, Destine J, Poirrier R. Midsaggital jaw movements as a sleep/wake marker. IEEE Trans Biomed Eng. 2009;56:303-9.

30. Senny F, Maury G, Cambron L, Leroux A, Destiné J, Poirrier R. The sleep/wake state scoring from mandibl movement signal. Sleep Breath 2011; (Epub).

31. De Chazal P, Heneghan C, McNicholas WT. Multimodal detection of sleep apnoea using electrocardiogram and oximetry signals. Phil Trans R Soc A. 2009;367:369-89.

32. Sommermeyer D, Schwaibold M, Schöller B, Grote L, Hedner J, Bolz A. Prediction of cardiovascular risk from peripheral pulse wave. IFMBE proceedings. World congress on Medical Physics and Biomed Eng. Vol 25/7. Springer Berlin Heidelberg, pp. 891-892 (2009)

33. Hedner J, White DP, Malhotra A, Hercovici S, Pittman SD, Zou D, et al. J Clin Sleep Med. 2011;7:301-6.

34. Schnall R, Shlitner A, Sheffy J, Kedar R, Lavie P. Periodic profound peripheral vasoconstriction - a new marker of obstructive sleep apnea. Sleep. 1999;22:939-46.

35. Dvir I, Adler Y, Freiark D, Lavie P. Evidence for fractal correlation properties in variations of peripheral arterial tone during REM sleep. Am J Physiol Heart Circ Physiol. 2002;283:H434-9.
36. Bar A, Pillar G, Dvir I, Sheffy J, Shlitner A, Lavie P. Evaluation of a portable device based on peripheral arterial tone for unattended home sleep studies. Chest. 2003;123:695-703.

37. Penzel T, Kesper K, Pinnow I, Becker HF, Vogelmeier C. Peripheral arterial tonometry, oximetry and actigraphy for ambulatory recording of sleep apnea. Physiol Meas. 2004;25:1025-36.

38. Guilleminault C, Connolly S, Winkle R, Melvin K, Tilkian A. Cyclical variation of the heart rate in sleep apnoea syndrome. Mechanisms, and usefulness of $24 \mathrm{~h}$ electrocardiography as a screening technique. Lancet. 1984;8369:126-31.

39. Somers VK, Dyken ME, Clary MP, Abboud FM. Sympathetic neural mechanisms in obstructive sleep apnea. J Clin Invest. 1995;96:1897-904.

40. Heneghan C, de Chazal P, Ryan S, Chua CP, Doherty L, Boyle P, et al. Electrocardiogram recording as a screening tool for sleep disordered breathing. J Clin Sleep Med. 2008;4:223-8.

41. Mendez MO, Bianchi AM, Mattteucci M, Cerutti S, Penzel T. Sleep apnea screening by autoregressive models from a single ECG lead. IEEE Trans Biomed Eng. 2009;56:2838-50.

42. Moody GB, Mark RG, Zoccola A, Mantero S. Clinical validation of the ECG-derived respiration (EDR) technique. Comput Cardiol. 1986;13:507-10.

43. Penzel T, McNames J, de Chazal P, Raymond B, Murray A, Moody G. Systematic comparison of different algorithms for apnoea detection based on electrocardiogram recordings. Med Biol Eng Comput. 2002;40:402-7.

44. • Kesper K, Canisius S, Penzel T, Ploch T, Cassel W. ECG signal analysis for the assessment of sleep-disordered breathing and sleep pattern. Med Biol Eng Comput 2011; (Epub). The retrospective study presents a new ECG-based analysis to detect sleep apnea and distinguishes slow wave sleep and REM sleep successfully.

45. Heneghan C, Chua CP, Garvey JF, de Chazal P, Shouldice R, Boyle $\mathrm{P}$, et al. A portable automated assessment tool for sleep apnea using a combined holter-oximeter. Sleep. 2008;31:1432-9.

46. Zamarron C, Gude F, Barcala J, Rodriguez JR, Romero PV. Utility of oxygen saturation and heart rate spectral analysis obtained from pulse oximetric recordings in the diagnosis of sleep apnea syndrome. Chest. 2003;12:1567-76.

47. Raymond B, Cayton RM, Chappell MJ. Combined index of heart rate variability and oximetry in screening for the sleep apnoea/ hypopnoea syndrome. J Sleep Res. 2003;12:53-61.

48. Fietze I, Penzel T, Alonderis A, Barbe F, Bonsignore MR, Calverly $\mathrm{P}$, et al. Management of obstructive sleep apnea in Europe. Sleep Med. 2011;12:190-7.

49. •- Pack AI. Sleep medicine: strategies for change. J Clin Sleep Med. 2011;7:577-9. The high prevalence and the cardiovascular consequences of sleep apnea require a change in the traditional sleep center based sleep medicine. Some services need to be done at home and others need to remain at hospitals. New concepts for organizing care in patients with sleep disorders are needed. A way for the development of such new concepts is proposed..

50. Penzel T, Fietze I, Hirshkowitz M, Vorstand der DGSM. Diagnostik in der Schlafmedizin. Somnologie. 2011;15:78-83.

51. - Skomro RP, Gjevre J, Reid J, McNab B, Ghosh S, Stiles M, et al. Outcomes of home-based diagnosis and treatment of obstructive sleep apnea. Chest. 2010;138:257-63. The study compares outcomes in terms of sleepiness and CPAP adherence over 4 weeks in patients with sleep apnea diagnosed and treated at home compared to the standard sleep center based approach. The completely different management approach is not inferior to the traditional sleep center based approach..

52. Kimoff RJ. To treat or not to treat: can a portable monitor reliably guide decision-making in sleep apnea. Am J Respir Crit Care Med. 2011;184:871-2. 